\title{
BACK CHIP TEMPERATURE IN ENVIRONMENTALLY CONSCIOUS TURNING WITH CONVENTIONAL AND INTERNALLY COOLED CUTTING TOOLS
}

\author{
Saiful Anwar Che Ghani ${ }^{1,2}$, Kai Cheng ${ }^{1}$ and Timothy Minton ${ }^{1}$ \\ ${ }^{1}$ Advanced Manufacturing and Enterprise Engineering Department \\ Brunel University, UB8 3PH, UK \\ Email: kai.cheng@brunel.ac.uk \\ ${ }^{2}$ Manufacturing Process Focus Group, \\ Faculty of Mechanical Engineering \\ Universiti Malaysia Pahang, 26600 Pekan, Pahang, Malaysia
}

\begin{abstract}
Central to machining processes is the interaction between the tool insert and the chip of material removed from the blank. Chip-insert interaction occurs when the chip slides on the rake face of the insert. Heat is generated by the friction inherent to this sliding process. The temperature in the cutting zone of both the insert and the chip rises, usually facilitating adhesion, diffusion, and more complex chemical and physical phenomena between the insert and the chip. These effects accelerate the insert wear, ultimately undermining the tool life. Thus, a number of methods have been developed to control heat generation. Most typically, metal working fluids are conveyed onto the rake face in the cutting zone. However, this solution may be not ideal from the point of view of cost, the environment, and contamination of the part, which may be unacceptable, for example, in healthcare and optical applications. In this study, microfluidic structures internal to the insert are examined as a means of controlling the heat generation.Conventional and internallycooled tools were compared in dry turning of AA6082-T6 aluminum alloy in two $3 \times 3$ factorial experiments of different machining conditions. Statistical analyses support the conclusion that chip temperature depends only on the depth of cut,and not on the feed rate or cutting speed. They also show that the benefit of cooling the insert internally increases as the depth of cut increases. Therefore, internallycooled tools can be particularly advantageous in roughing operations.
\end{abstract}

Keywords: Internally cooled cutting tool, environmentally conscious machining, back chip temperature.

\section{INTRODUCTION}

The high levels of heat that are generated in and around the cutting zone during machining operations are detrimental to the performance of the cutting tool. For instance, the generated heat can have a serious impact on the quality of the machined parts, causing potential scenarios where re-work or even scrapping of the part is required. The notion of dousing the cutter and work-piece with cooling fluid was first reported as a beneficial technique by Taylor (1907), who proposed that the cutting zone requires a flood of coolant to manage the increase in temperature at the tool-chip interface. The compositions of modern metal working fluids are complex and require complicated collection-disposal-treatment regimes that can be costly. Many metal 
working fluids are hazardous to the operator and can cause respiratory and dermatological illnesses (Bernstein, Lummus, \& Santilli, 1995). Many studies have been carried out based on statistics that highlight that between $7 \%$ and $17 \%$ of the cost of a manufactured part is related to the coolant and the associated treatment activities that surround it (Klocke \& Eisenblaetter, 1997; Kadirgama, Rahman, Ismail, \& Bakar, 2011). A significant effort has been made to remove the coolant completely (dry machining) (Klocke \& Eisenblaetter, 1997; Weinert, Inasaki, \& Sutherland, 2004), or to just reduce the amount used (Medium Quantity Lubrication) (Sreejith \& Ngoi, 2000; Najiha, Rahman, Yusoff, \& Kadirgama, 2012a; Najiha, Rahman, Kamal, Yusoff, \& Kadirgama, 2012b). Removing flood cooling is beneficial to the environment and to the machine operator's health, while it also provides a financial advantage for the manufacturer. However, the fundamental machining requirement of managing the temperature in the cutting zone must be confronted. The potential hazards associated with high cutting temperatures can include tool failure, slowed production rates, and geometrical inconsistency of the machined part leading to higher production costs (Byrne, Dornfeld, \& Denkena, 2003). The desirable result of the removal of cutting fluids from the metal-cutting process is the opportunity to carry out contamination-free machining. There may be applications where an external coolant supply has prohibitive constraints. For example, cases when machining sensitive materials for optics or biomedical applications, or when machining harmful work-pieces such as radioactive materials. Avoiding the use of external coolant when cutting radioactive materials would reduce the potential for further contamination. The aim of this investigation is to compare dry contamination-free machining, both with a conventional and with an internally cooled tool, to address the issue of raised cutting temperatures. A large amount of heat is generated at the tool-chip interface from the shearing of material by the cutting tool (Longbottom \& Lanham, 2005). Numerical models and simulations suggest that temperatures at this interface can be as high as $400{ }^{\circ} \mathrm{C}$ when machining aluminum alloys (Lazoglu \& Altintas, 2002; Dinc, Lazoglu, \& Serpenguzel, 2008). At these temperatures, specific microstructural changes occur. It has been documented that between $60 \%$ to $95 \%$ of this heat is dissipated within the chips formed by the cutting process (Quan, He, \& Dou, 2008). Some heat is transferred to the cutting tool when hot chips slide over the rake face, which causes wear via diffusion and eventually creates a crater (Spitler, Lantrip, \& Nee, 2003). High temperatures in this region also create a rapid formation of a Built-Up-Edge (BUE), where the work-piece material adheres to the surface of the tool making it blunt. The BUE affects the quality of the part, and it increases forces and the cutting temperature. Therefore, the high temperature within the interface region must be controlled.

A solution to this age-old problem is the notion of an internally supplied coolant within a closed loop system. This was described first in the seventies (Jeffries \& Zerkle, 1970; Jeffries, 1972) and has been attempted many times subsequently, using a plethora of different designs and methods (Zhao, Barber, \& Zou, 2006; Rozzi, Sanders, \& Weibo, 2011; Sanchez, Scalon, \& Abreu, 2011). Fundamentally, a fluid is pumped through the tool shank into a heat exchanger module situated beneath the cutting insert and back out through the tool shank. The main premise of this system is to cool the cutting zone by the internally conveyed fluid media. It may seem counter intuitive to channel heat into the tooling zone owing to the potential problems that this may cause (higher wear rates, diffusion, adhesion, tool expansion). However, the internal fluid will facilitate heat transfer from the cutting zone to an external heat sink much more quickly than the time needed for these potential problems to arise within the tooling. The 
primary intention when cooling the cutting zone is to extend the useful life of a cutting insert. The rationale of this is quite simple; the hotter the tool, the faster it wears out (Spitler et al., 2003). Without an external supply of coolant, this wear is rapid (Taylor, 1907). However, by cooling the cutter, the increased thermal gradient results in greater heat transfer from the cutting zone, which allows prolonged cutter life; thus, increasing productivity whilst maintaining product quality.

There are many difficulties in measuring the temperature within the cutting zone. Many authors have attempted this using an array of different techniques. The most popular method is by use of the embedded thermocouple (Longbottom \& Lanham, 2005). This would be easy to implement using a standard monolithic cutting insert. However, in this investigation, the internal geometry of the cooling structure precludes this option. The thermocouple is required to be as close to the cutting tip as possible. However, the cooling channels are also required to be as close to the cutting tip as possible in order to maximize the effect of the internal coolant. Clearly, both cannot occupy the same space. Therefore, preference has been allocated to the cooling channels. There is a very large thermal gradient experienced during cutting and a thermocouple placed more than a few millimeters from the tool tip will not give an accurate measurement of the cutting temperature. Another circumstance that deters investigators from using embedded thermocouples is the change in the dynamics of heat transfer within the tool inserts caused by the thermocouple itself and the necessary hole (Longbottom \& Lanham, 2005). Another method that has been used in previous studies is the tool-work thermocouple or dynamic thermocouple (O'Sullivan \& Coterell, 2001). This is difficult to use because of the relatively low electrical conductivity of the tungsten carbide inserts. In this study, a non-contact infrared pyrometer is used to measure the back-chip temperature. This method has been used successfully in previous studies to validate the numerical and finite element analysis models where the tool-chip interface temperatures have been calculated (Davies, Ueda, \& M'Saoubi, 2007). This method is deemed difficult to use owing to the fluctuating emissivity of materials such as metals. However, once the pyrometer has been calibrated correctly by using the black body technique, the measurements can be considered reliable.

\section{EXPERIMENTAL DETAILS}

The intention of testing dry cutting conditions led to the selection of a material for the test parts that can facilitate cutting operations. An aluminum alloy was considered because its characteristics of machinability make the wear resistance properties of the insert, which is the central critical part of the setup, less demanding. Therefore, a reduced number of conventional and internally cooled inserts was needed than would have been required otherwise, if machining another material, e.g., steel. The aluminum alloy AA6082-T6 with relatively high silicon and magnesium content (0.7-1.3 and 0.6$1.2 \%$ in weight, respectively) was selected owing to its ready availability and widespread use in several applications. One cylindrical bar was machined in cutting trials with a conventional tool insert and a second bar was machined in cutting trials with the internally cooled tool. The diameter and length of the two AA6082-T6 blanks were 65 and $450 \mathrm{~mm}$. The length of the bar was sufficient to observe, in each trial, the establishment of a steady measured temperature of the back-chip surface. A minor drawback was that the high aspect ratio of the blank required a tailstock to be used on the lathe. The cutting trials were performed using an Alpha Colchester Harrison 600 Group CNC lathe. In Figure 1, the machine setup is displayed. In the figure, the tool 
system, the work-piece, and the pyrometer used to measure the back-chip temperature are shown.

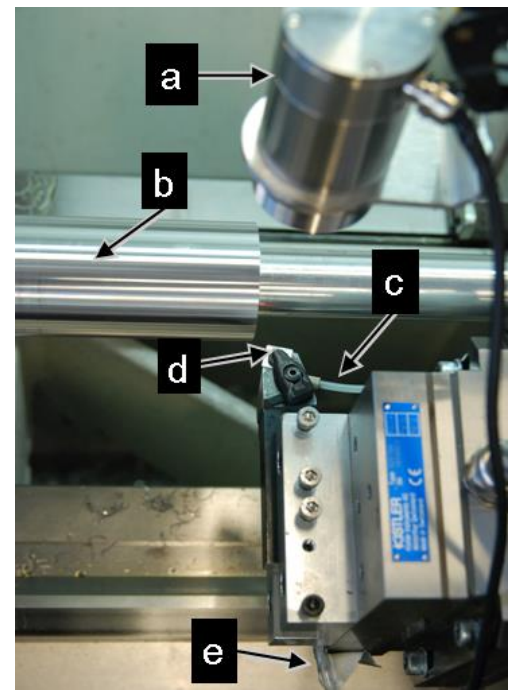

Experimental setup

a: pyrometer

b: AA6082- T6 aluminum alloy blank

c: outlet tube for internal cooling fluid

d: cutting insert

e: inlet tube for internal cooling fluid

Cutting parameters

Cutting speed, $v[\mathrm{~mm} / \mathrm{min}]: 250,300$, and 350

Feed rate, $f[\mathrm{~mm} / \mathrm{rev}]: 0.10,0.15$, and 0.2

Depth of cut, $d[\mathrm{~mm}]: 0.20,0.35$, and 0.5

Figure 1.Experimental setup of the CNC lathe.

Internally cooled tools were designed and manufactured by modifying commercially available tools. This approach reduced both the development time and cost significantly. The designed internally cooled cutting tool comprises three main components: (a) the modified cutting insert, (b) the cooling adapter accommodating the micro channel, and (c) the tool holder with the inlet and the outlet ports. The selected cutting insert was square-shaped, without a chip-breaker and made of tungsten carbide with $6 \%$ cobalt (SNUN120408, produced by Hertel). The insert was machined by electro-discharge machining to fabricate a square bottle-cap-shaped part with a wall thickness of $1 \mathrm{~mm}$ (Khan, Rahman, Kadirgama, Maleque, \& Ishak, 2011; Singh \& Singh, 2011). It was then attached to the cooling adapter such that an empty cavity was created near the cutting zone between the insert and the adapter. This cavity hosts the flow of coolant during cutting operations. The cooling adapter was machined on a fiveaxis micro-milling machine to accommodate ad hoc designed micro channels of $800-\mu \mathrm{m}$ diameter, which enable coolant recirculation inside the above-mentioned cavity. The module made of the insert and the cooling adapter was then assembled with a tool holder. An off-the-shelf tool holder (CSBNR 2525M 12-4, produced by Sandvik) was modified such that inlet and outlet tubes for the internal coolant could be fixed on the bottom and side surfaces, respectively.

Figure 2 displays a production phase of the cooling adapter on the five-axis micro-milling machine, the square bottle-cap insert, the cooling adapter, and the assembled tool system. The coolant used in the experiment was pure water with corrosion inhibitor, which was pumped in a closed loop system by a micro diaphragm liquid pump (NFB 60 DCB made by KNF- Neuberger). The pump can deliver up to $1.2 \mathrm{l} / \mathrm{min}$ with twin heads, which allows the coolant speed to be varied and controlled. In this study, for simplicity, the coolant speed was kept constant at $0.3 \mathrm{l} / \mathrm{min}$. 


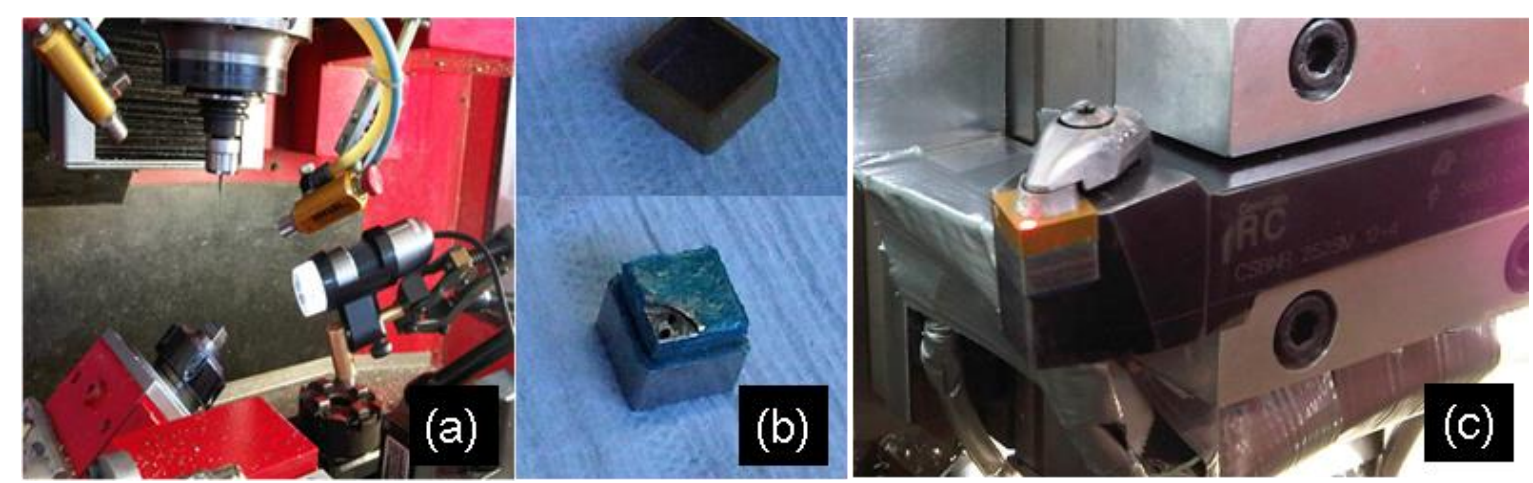

Figure 2. (a) Fabrication of the cooling adapter on the 5-axis Kern machining center; (b) the square bottle-cap-shaped cutting insert on the top, and the cooling adapter at the bottom; (c) the ready-to-use internally cooled cutting tool and protected lubricating fluid tubes.

The experimental setup was complemented by a thermal sensor, data acquisition system, and data processing software. The thermal sensor utilized in this study was a laser pyrometer with a minimum spot size of the beam equal to $0.45 \mathrm{~mm}$ ( $\mu$-Epsilon, model CTLM3- H1 CF2). The pyrometer was fixed at $150 \mathrm{~mm}$ above the cutting insert and pointed to a single point on the insert's rake face, about $1 \mathrm{~mm}$ from the cutting edge (Figure 1). The temperature measurements were acquired at $1000 \mathrm{~Hz}$ and then transferred to a computer-based acquisition system by serial cable. Dedicated software (Compact Connect) was provided by the pyrometer manufacturer, which enabled the measured temperatures to be displayed dynamically. Additionally, within this software, the emissivity of the target surface could be set. In this study, the emissivity of the target surface was determined as 0.78 . The values of the instantaneous chip temperatures recorded during all the cutting trials appeared to achieve a steady-state condition. In an attempt to identify this state unambiguously, a procedure was devised. A unique temperature value was associated with the identified steady state for each cutting trial. In this way, high reproducibility of the steady-state temperature is assured. The procedure involved three stages. First, the start and the end of each machining operation were identified uniquely by recording the instants when the measured temperature exceeded the minimum temperature measurable by the pyrometer $\left(150{ }^{\circ} \mathrm{C}\right)$. Second, the interquartile range of the temperature distribution measured between the identified start and end was calculated. Third, the average of the temperatures measured within the interquartile range was computed and defined as the steady-state temperature of the chip. Figure 3 illustrates this procedure. The steady-state chip temperature measured during the machining trials is taken as representative of all the thermal information available regarding the trial performed under pre-specified, designed machining conditions. 

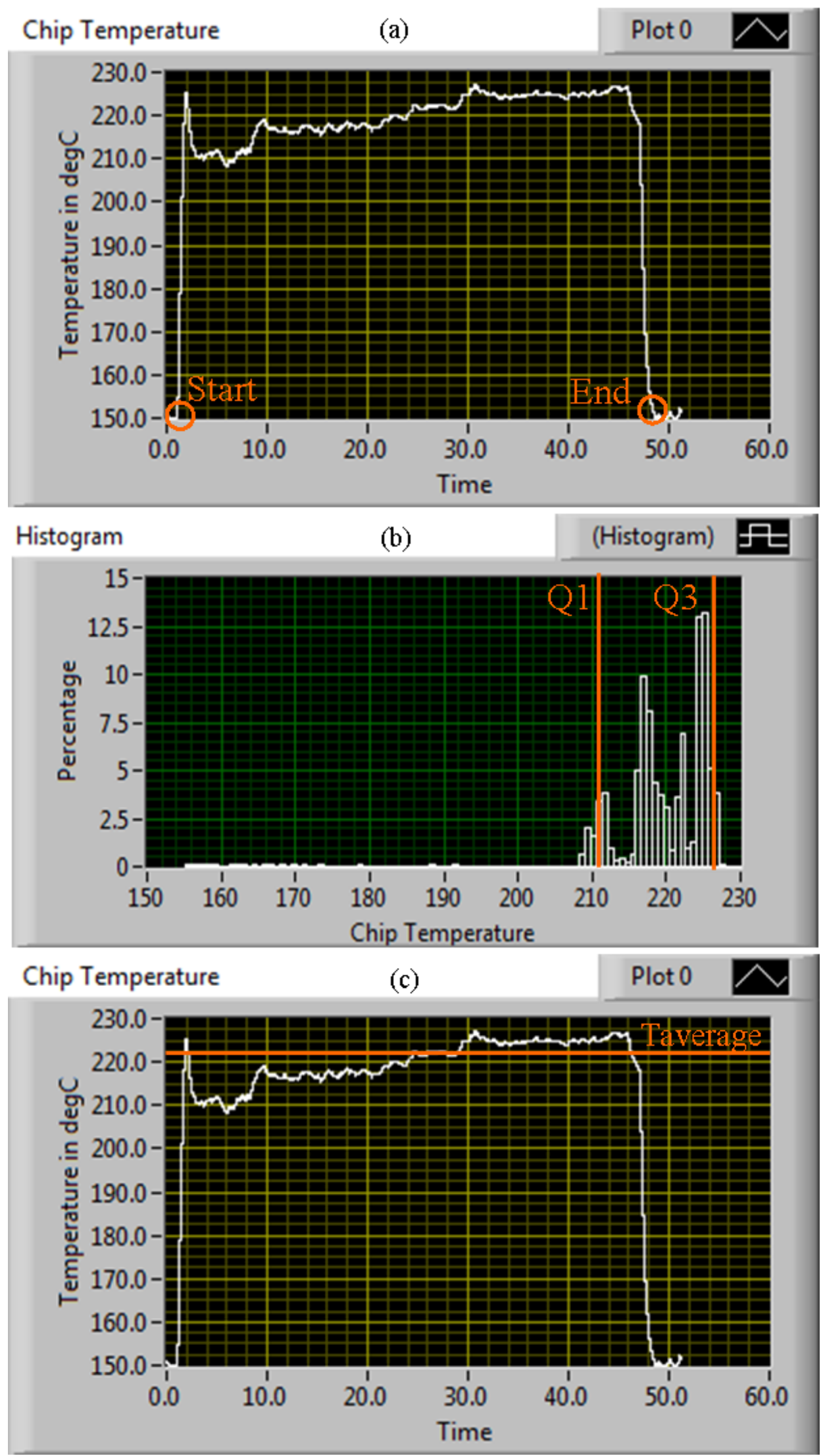

Figure 3.Three stages of the procedure to determine the steady-state cutting temperature. 


\section{RESULTS AND DISCUSSION}

During machining operations with the internally cooled tool, the temperature $(T)$ of the chip was measured under a set of experimental conditions identified by three controllable technological variables: the depth of cut $(d)$, the feed rate $(f)$, and the cutting speed $(v)$. Owing to their numerical nature, these variables have been considered as continuous rather than categorical. For each variable, three values were considered as identifying a region of interest in the space $(d, f, v)$. Symbols, units and values of the technological variables are presented in Figure 1. The number of the different experimental conditions (treatments) was therefore $3 \times 3$, i.e., 27 . For each of the 27 treatments, the cutting test was replicated three times; thus, giving a total of 81 tests. The sequence of the treatments was generated by assigning each of them a unique label and then by drawing a sequence of 27 labels randomly from the possible 27! label permutations. Changing from one experimental condition to another was a timeconsuming operation that prohibited randomizing fully the order of the 81 tests. If some nuisance event occurred while performing the three tests for a specific treatment (e.g., a surge of the laboratory temperature), then its potential effect on the chip temperature would have been attributed erroneously to the treatment. However, the controlled conditions of the laboratory where the tests were performed limited the likelihood of the occurrence of such random nuisance events. The main objective of this investigation is to construct a quantitative functional relationship (i.e., a model) between $T$ and $(d, f, v)$ in a region of the three-dimensional technological space $(d, f, v)$. The intended aim of the model is to explain quantitatively the relationship between the chip temperature $T$ and the technological variables $(d, f, v)$. The model could then be also used to predict the expected chip temperature for a given technology (i.e., for a given triplet $(\bar{d}, \bar{f}, \bar{v})$ ). Because all the variables are continuous, the statistical linear model fitted to the data is usually referred to as a regression model (a more detailed explanation is given by Faraway (2005)).

In Figure 4, the 81 test results are grouped by depth of cut. To make the figure clearer, overlapping points at the same depth of cut were separated by adding a random small amount to each abscissa of the points (a procedure called jittering, (Crawley, 2012)). The average chip temperature for each depth of cut is designated with a triangle, whereas the median is depicted by a square. The graph suggests that the chip temperature increases linearly with depth of cut, and the boxplot with notches, shown in the same figure, confirms this interpretation of the graph. For each group of data (i.e., for the $T_{\mathrm{s}}$ measured at one of the three depths of cut), each box comprises three horizontal segments and each of these segments identifies the first quartile, the median, and the third quartile of the $T_{\mathrm{s}}$. Two notches (i.e., two vertical ' $v$ ' with apexes ending on the median segment) are calculated and drawn on each side of the boxes. If the notches of two boxes do not overlap, the medians of the two groups are significantly different at about the 95\% confidence level (McGill, Tukey, \& Larsen, 1978). The dashed vertical lines in the same figure (called whiskers) extend 1.5 times the value of the interquartile range. They are meant to identify values lying far apart from the majority of the data in a group. In Figure 4, it is noticed that there are three such points when $d=0.5 \mathrm{~mm}$. A further investigation did not highlight any assignable cause for the occurrence of these values. Therefore, these three experimental results were treated as unlikely but possible events and were not excluded from the analysis. 


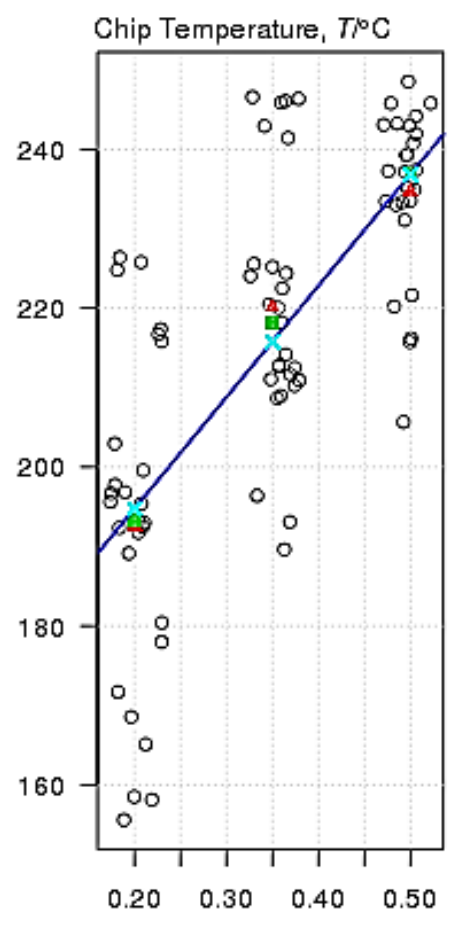

(a) Depth, $d / \mathrm{mm}$

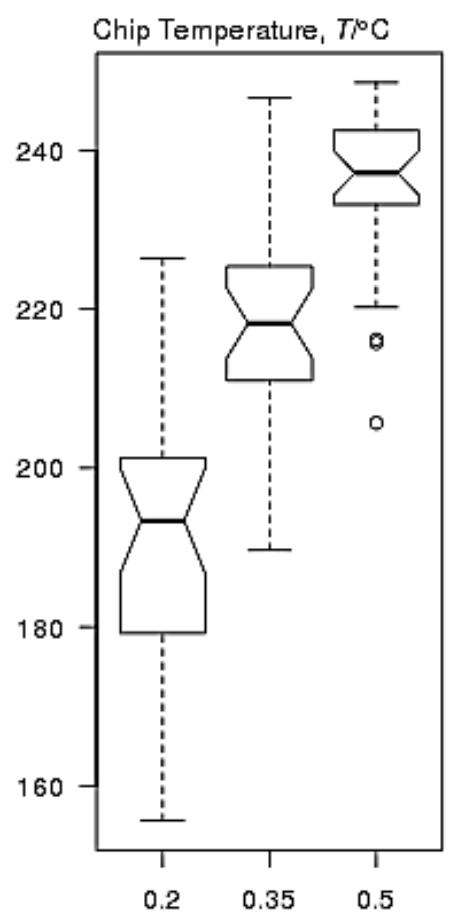

(b) Depth, d/mm

Figure 4. (a) Chip temperature verses depth of cut and (b) the boxplot representation of the relationship.

In Figure 5, the chip temperature data have been grouped by feed rate and by cutting speed. In contrast to Figure 4, these boxplots do not suggest any significant difference in the median chip temperature when the feed rate is changed. Similarly, changing the cutting speed does not appear to affect $T$ significantly. Five extreme points appear in the two boxplots of Figure 5. However, as before, further investigation did not reveal any assignable cause for these points lying far from the majority of values of chip temperatures for their groups; thus, these points were not excluded from the analysis. In the same figure, it can also be seen that the variability of chip temperature is dubiously constant. In particular, the group for a feed rate of $0.1 \mathrm{~mm} / \mathrm{rev}$ is difficult to interpret because of the simultaneous presence of the three extreme points and a noticeably small interquartile range in comparison with the other groups. Interaction plots were also examined, but they did not exhibit any pattern that would lead to conclude in favor or in denial of a significant second-order interaction effect of $(d, f, v)$ on $T$. To select a model to fit the data, a stepwise procedure with forward selection of the independent variables was followed (Faraway, 2005). Starting from the model with no variables included (also known as the null model) and consisting of only the mean chip temperature, models with increasing complexity are considered by adding one variable at a time. When a variable is included, the p-value of the F-test assessing the significance of the decrease in deviance yielded by the inclusion is evaluated. Should the reduction of deviance (i.e., the sum of the squares of the residuals, alias the unexplained variation in the response variable) be statistically significant, then the corresponding technological variable is included in the model, otherwise it is not (Crawley, 2012). Second-degree terms of two variables are included in the model only if each of the single variables is significant per se. This decision is consistent with the fact that the model is expected not to depend on 
the arbitrary choice of the origin or on the orientation of the axes in the space of the independent variables (Venables, Ripley, \& Venables, 1994) about marginality restrictions (Faraway, 2005). The results of the variable selection procedure are shown in Table 1. The calculations were performed with $\mathrm{R}$, a language and environment for statistical computing. As shown in Table 1, the feed rate and the cutting speed do not contribute significantly to reducing the variability not explained by the model. This happens when $f$ and $v$ are added to the null model separately (p-values 63.9\% and $45.4 \%$, respectively, in Table 1) and when they are added to the model that already includes $d$ (p-values $49.77 \%$ and $27.90 \%$ for $f$ and $v$, respectively, in Table 1).

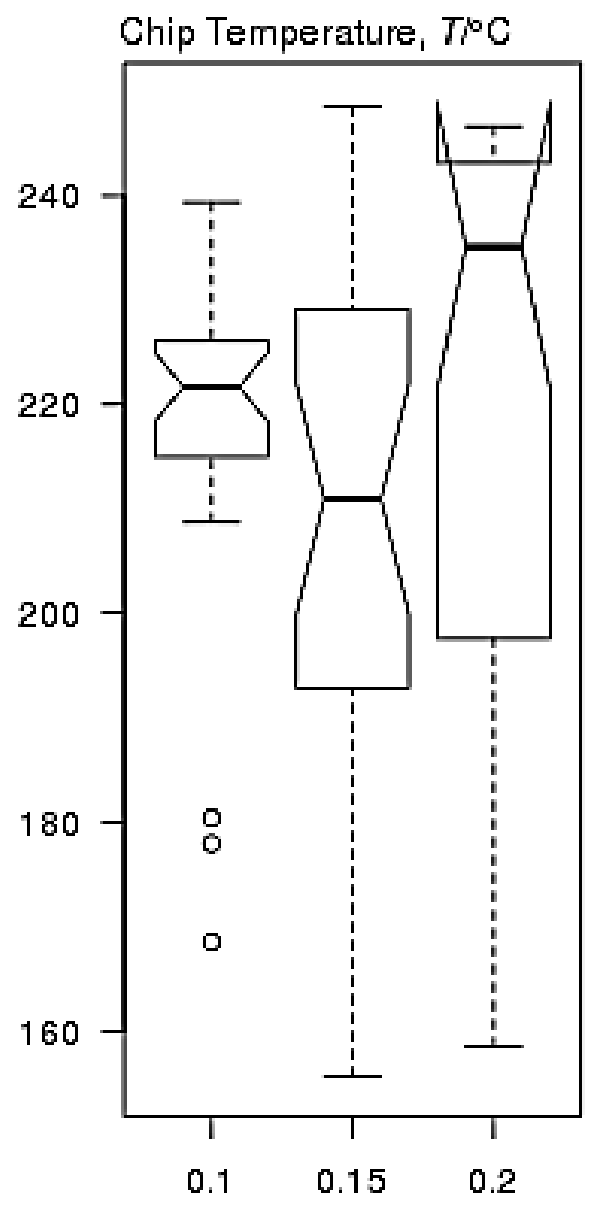

(a) Feed rate, $d / \mathrm{mm}^{\mathrm{rev}}{ }^{-1}$

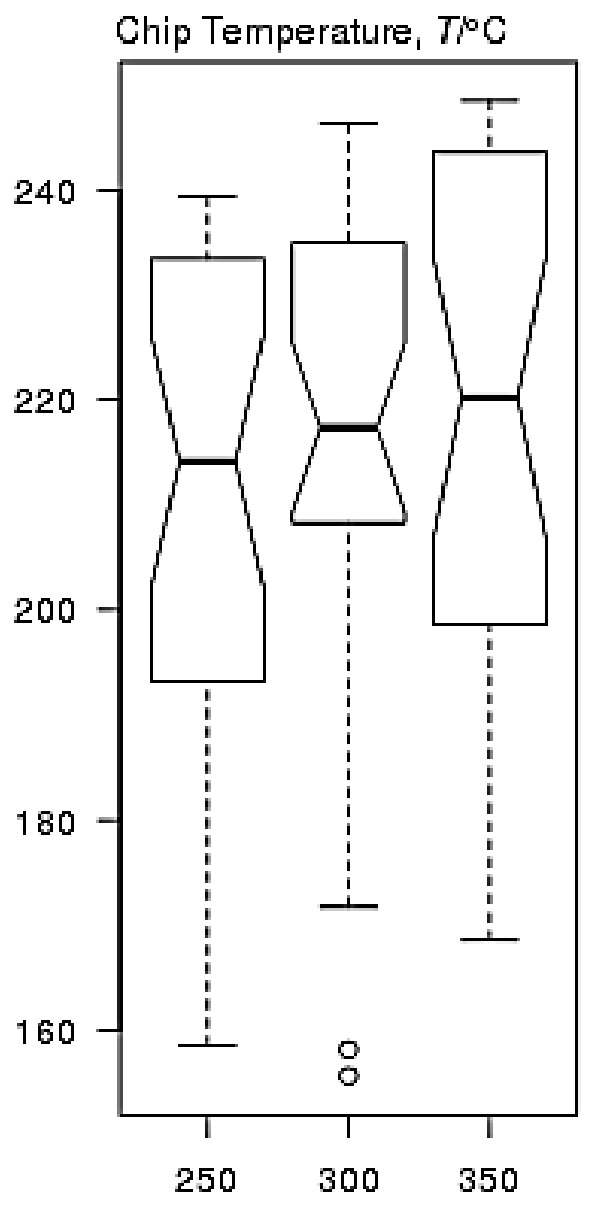

(b) Cutting speed, $d / \mathrm{mm} \mathrm{min}^{-1}$

Figure 5. Boxplot of the back chip temperature grouped by: (a) feed rate and (b) cutting speed.

The feed rate and the cutting speed are therefore not included in the proposed statistical model, which is synthesized by the following equation:

$$
T_{i}=\beta_{0}+\beta_{1} d_{i}+\epsilon_{i}
$$

Equation (1) describes the expected chip temperature versus the depth of cut as a straight line. The symbols $\beta_{0}$ and $\beta_{1}$ are two parameters that represent the intercept and 
the slope of the line, respectively. The index $i=1,2 \ldots, 81$ identifies each of the actual cases in the available data. The terms $\epsilon_{i}$ are random variables that without losing generality are assumed to be independently and identically distributed with mean zero and constant variance $\sigma^{2}$. If they are also normal, further analysis of the model parameters is facilitated. The $2+1$ parameters $\left(\beta_{0}, \beta_{1}\right.$, and $\left.\sigma\right)$ have been estimated with the ordinary leastsquares method (OLS) using the functions available in $\mathrm{R}$ (RDevelopment Core Team, 2011). A more complex model, including a quadratic term in the depth of cut, was also considered, namely:

$$
T_{i}=\beta_{0}+\beta_{1} d_{i}+\beta_{1} d_{i}^{2}+\epsilon_{i}
$$

Table 1. Selection of the technological variables to include in the model (Df: degrees of freedom, Sum Sq: sum of squares, Mean Sq: mean of squares).

\begin{tabular}{lrrrrr}
\hline & Df & Sum Sq & Mean Sq & F-value & p-value \\
\hline Depth & 1 & 23927 & 23927.0 & 87.72 & 0.0000 \\
Residuals & 79 & 21548 & 272.8 & & \\
Feed & 1 & 127 & 127.46 & 0.22 & 0.6388 \\
Residuals & 79 & 45347 & 574.01 & & \\
Speed & 1 & 323 & 323.34 & 0.57 & 0.4542 \\
Residuals & 79 & 45151 & 571.53 & & \\
Depth & 1 & 23927 & 23927 & 87.13 & 0.0000 \\
Feed & 1 & 127.46 & 127.46 & 0.46 & 0.4977 \\
Residuals & 78 & 21420 & 274.62 & & \\
Depth & 1 & 23927 & 23927 & 87.93 & 0.0000 \\
Speed & 1 & 323.34 & 323.34 & 1.19 & 0.2790 \\
Residuals & 78 & 21224 & 272.10 & & \\
\hline
\end{tabular}

As shown in

Table 2, the model with the second-degree term in d (i.e., $d^{2}$ ) does not lead to a significant reduction of the unexplained variation of the response, when compared with the simpler model of a straight line ( $\mathrm{p}$-value $=9.6 \%$ ). The introduction of $d^{2}$ in Eq. 1 would not improve the fitting of the model to the data and therefore, would be unjustified. The availability of replicated tests for each of the depths of cut considered allows the experimenter to test the model for lack of fit (Faraway, 2005). If the model of Eq. 1 is of the wrong form in $d$ or too simple (i.e., too few parameters), then the OLS estimate $\hat{\sigma}$ would overestimate $\sigma$. If it were too complex (i.e., with too many parameters), $\hat{\sigma}$ would underestimate $\sigma$. The model that yields the smallest estimate of $\sigma$ is the model that would fit a parameter for each group of depth of cut (also known as the saturated model, or in this case, the one-way analysis of variance model) $\sigma$ for such a model would represent the variability of $T$ around the mean chip temperature of a group when the data are grouped by depth of cut. From a different perspective, for a saturated model, $\hat{\sigma}$ represents the estimated repeatability standard deviation of the measuring procedure for the chip temperature. If the model of Eq. 1 does not exhibit a $\hat{\sigma}$ significantly larger than the estimated repeatability standard deviation, then the assumption that the model of Eq. 1 fits the data cannot be rejected. The results of the tests are displayed in Table 3. 
Table 2. Comparison of first and second-degree models (Res Df: residuals degrees of freedom, RSS: residuals sum of squares).

\begin{tabular}{llllclc}
\hline & Res. Df & RSS & Df & Sum of Sq & F-value & p-value \\
\hline 1 & 79 & 21548 & & & & \\
2 & 78 & 20792 & 1 & 755.64 & 2.83 & 0.0962 \\
\hline
\end{tabular}

Table 3. Test for lack of fit (first row, regression model; second row, saturated model).

\begin{tabular}{lllllll}
\hline & Res. Df & RSS & Df & Sum of Sq & F-value & p-value \\
\hline 1 & 79 & 21548 & & & & \\
2 & 78 & 20792 & 1 & 755.64 & 2.83 & 0.0962 \\
\hline
\end{tabular}

A p-value $=9.62 \%$ suggests that there is not a significant lack of fit for the model of Eq. (1). Also, from Table 3, the estimated repeatability standard deviation of the chip temperature measuring procedure is equal to $16.3{ }^{\circ} \mathrm{C}$, i.e., $\sqrt{(20792 / 78)}{ }^{\circ} \mathrm{C}$, whereas the estimated standard deviation of the errors for the model of Eq. 1 is $16.5^{\circ} \mathrm{C}$, i.e., $\sqrt{(21548 / 79)}{ }^{\circ} \mathrm{C}$. In Figure 4, the OLS straight line is displayed. The expected values of the chip temperature for the depths of cut tested experimentally are also shown (they are designated with an $\mathrm{x}$ in Figure 4). Graphically, the test for lack of fit in Table 3 corresponds to having ascertained the non-significance of the difference between the fitted (the $\mathrm{x}$ points) and the mean points (the triangular points) for each of the cutting depths tested. The estimated $\hat{\beta}_{0}$ and $\hat{\beta}_{1}$ of $\beta_{0}$ and $\beta_{1}$ (i.e., the estimated intercept and slope) are displayed in Table 4, together with their estimated standard deviations (i.e., standard errors).

Table 4.OLS estimations of the parameters in the linear model with constant error variance.

\begin{tabular}{llll}
\hline Parameter & Lower & Estimate & Upper \\
\hline $\boldsymbol{\beta}_{\mathbf{0}} /{ }^{\circ} \boldsymbol{C}$ & 155.6 & 166.7 & 177.7 \\
$\boldsymbol{\beta}_{\mathbf{1}} /{ }^{\circ} \boldsymbol{C} \mathbf{~ m m}^{\mathbf{1}}$ & 110.5 & 140.3 & 170.1 \\
$\boldsymbol{\sigma} /{ }^{\circ} \boldsymbol{C}$ & 14.29 & 16.52 & 19.56 \\
\hline
\end{tabular}

The hypothesis that the errors $\epsilon_{i}(\mathrm{i}=1,2, \ldots, 81)$ are independent, identically distributed random variables with constant variance $\sigma^{2}$ is graphically tested on the residuals, i.e., the experimental data minus the corresponding predictions of the model. If the errors are independent with equal variance, then the residuals should not exhibit any pattern or any varying scatter, regardless of how they may have been grouped. In Figure 6 , the residuals are displayed versus the values fitted by the model of Eq. 1 versus the depth of cut. The quantities on the $\mathrm{x}$-axis have been jittered for clarity of the figures. The averages of the residuals having the same abscissa are also displayed as triangular points (there are 3 groups of 27 data points in each of the two diagrams). 


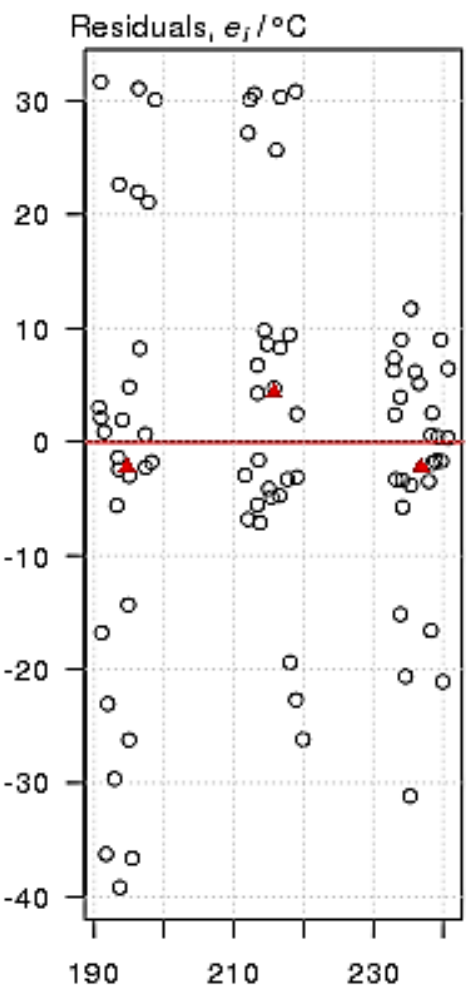

(a) Fitted values, $\tilde{T} /{ }^{\circ} \mathrm{C}$

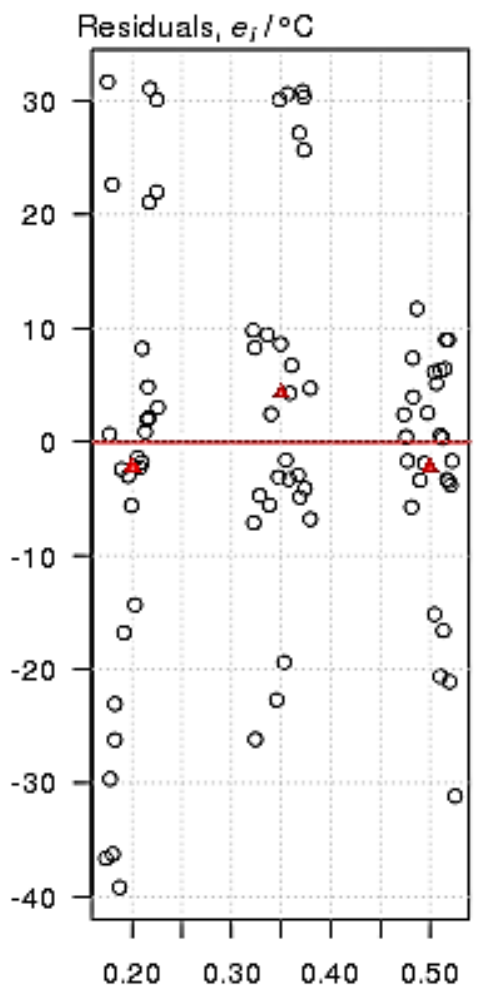

(b) Depth, d/mm

Figure 6. Residuals of the model in Eq. 1 against: (a) fitted values and (b) depth of cut.

Figure 6 raises the suspicion that the variance of the errors is not constant. In particular, the residuals with lower fitted values appear to have larger dispersion than those identified by a larger fitted temperature. Because the fitted temperature is proportional to the depth of cut, according to Eq. 1, it is not surprising that in Figure 6 the residuals appear to have lower variation when the depth of cut is larger. Based on this observation, new models were considered to account for the heteroscedastic errors by using the classes of variance functions provided in the $\mathrm{R}$ package nlme (Pinheiro, Bates, \& DebRoy, 2007) and described in chapter 5 of Pinheiro and Bates (2000). The variance function that offered the best fitting of the model to the data in terms of the Akaike Information Criterion (AIC), as described by Pinheiro and Bates (2000), was the exponential variance function (i.e., $\operatorname{varExp}())$. The variance of the errors is modeled by this class, as described in the following equation:

$$
\operatorname{Var}\left(\epsilon_{i}\right)=\sigma^{2} e^{2 \delta d_{i}}
$$

In Eq. (3), $\sigma$ and $\delta$ are two parameters in the model that are estimated by using the restricted maximum likelihood method (reml) as implemented in the gls() function of nlme. The estimates are displayed in Table 5, together with approximate 95\% confidence intervals for the same estimated parameters. 
Table 5.REML estimations of the parameters in the extended linear model with error variance varying exponentially.

\begin{tabular}{llll}
\hline Parameter & Lower & Estimate & Upper \\
\hline $\boldsymbol{\beta}_{\mathbf{0}} /{ }^{\circ} \boldsymbol{C}$ & 158.1 & 170.5 & 182.9 \\
$\boldsymbol{\beta}_{\mathbf{1}} /{ }^{\circ} \boldsymbol{C} \mathbf{~ m m}^{-\mathbf{1}}$ & 101.5 & 130.4 & 159.2 \\
$\boldsymbol{\delta}$ & -3.6588 & -2.3087 & -0.95858 \\
$\boldsymbol{\sigma} /{ }^{\circ} \boldsymbol{C}$ & 21.03 & 34.57 & 56.84 \\
\hline
\end{tabular}

A comparison between Table 4 and Figure 6 raises the suspicion that the variance of the errors is not constant. In particular, the residuals with lower fitted values appear to have larger dispersion than those identified by a larger fitted temperature. Because the fitted temperature is proportional to the depth of cut, according to Eq. 1, it is not surprising that in Figure 6 the residuals appear to have lower variation when the depth of cut is larger. Based on this observation, new models were considered to account for the heteroscedastic errors by using the classes of variance functions provided in the R package nlme (Pinheiro, Bates, \& DebRoy, 2007) and described in chapter 5 of Pinheiro and Bates (2000). The variance function that offered the best fitting of the model to the data in terms of the Akaike Information Criterion (AIC), as described by Pinheiro and Bates (2000), was the exponential variance function (i.e., varExp()). The variance of the errors is modeled by this class, as described in the following equation:

$$
\operatorname{Var}\left(\epsilon_{i}\right)=\sigma^{2} e^{2 \delta d_{i}}
$$

In Eq. (3), $\sigma$ and $\delta$ are two parameters in the model that are estimated by using the restricted maximum likelihood method (reml) as implemented in the gls() function of nlme. The estimates are displayed in Table 5, together with approximate 95\% confidence intervals for the same estimated parameters.

Table 5 reveals of no significant difference of both intercept and slope between the model of Eq. 1 and the model with the variance of the errors described by Eq. (3). Similar analyses have been performed on experimental data for machining with an uncooled tool. These analyses led to the same conclusion, which was that only the depth of cut is a significant explanatory variable for the back chip temperature. The fitted model can be described with the same terms present in Eq. (1). The OLS estimates of the model parameters and their 95\% confidence intervals, under the hypothesis of normally distributed errors, are displayed in Table 7. In contrast to the case of the cutting trials with the internally cooled tool, in this model, the graphical analyses of realized residuals do not suggest any violation of the hypothesis of independent, identically distributed and homoscedastic errors. In particular, in Figure 8, the residuals do not exhibit any pattern of variance increasing with the depth of cut, as was instead the case for the internally cooled tool (Figure 6). 


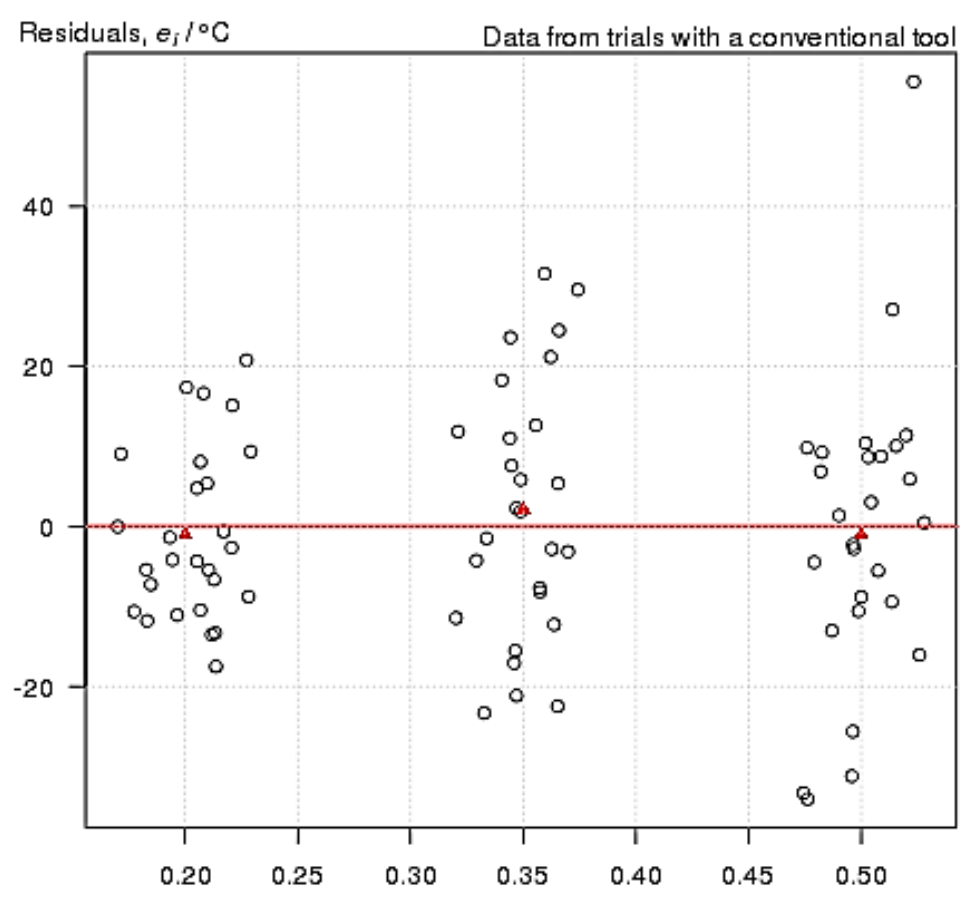

(b) Depth, $d / \mathrm{mm}$

Figure 7. Residuals against depth of cut for the model fitted to the data from the trials with the uncooled tools.

A possible reason of this discrepancy between Figure 6 and Figure 7 can be identified in the measuring system of the chip temperature. The diameter of the pyrometer laser beam was in fact $0.45 \mathrm{~mm}$. Therefore, when measuring the chip temperature, if the width of the chip is smaller than $0.45 \mathrm{~mm}$, the part of the laser beam that exceeds the size of the chip may hit upon part of the tool rake face, which is expected to be slightly hotter than the chip.

Table 6. OLS estimations of the parameters in the linear model fitted to the data from the trials with the uncooled tools.

\begin{tabular}{llll}
\hline Parameter & Lower & Estimate & Upper \\
\hline $\boldsymbol{\beta}_{\mathbf{0}} /{ }^{\circ} \boldsymbol{C}$ & 134.1 & 144.5 & 154.9 \\
$\boldsymbol{\beta}_{\mathbf{1}} /{ }^{\circ} \boldsymbol{C} \mathbf{~ m m}^{-1}$ & 199.6 & 227.6 & 255.7 \\
$\boldsymbol{\sigma} /{ }^{\circ} \boldsymbol{C}$ & 13.45 & 15.54 & 18.40 \\
\hline
\end{tabular}

It may be argued that if this interpretation holds, then for a depth of cut less than $0.45 \mathrm{~mm}$, the chip temperature measurements may be biased due to the effect of the potential exposure of the rake face to the laser beam. This consideration may also help to explain the rather unexpected circumstance that at $d=0.20 \mathrm{~mm}$, six measured chip temperature values obtained with the internally cooled tool are higher than any other temperature measured, when machining at the same depth of cut with a conventional tool (Figure 8). In addition, this potential bias in measuring the temperature may be the reason why the straight lines in Figure 8 intersect. 


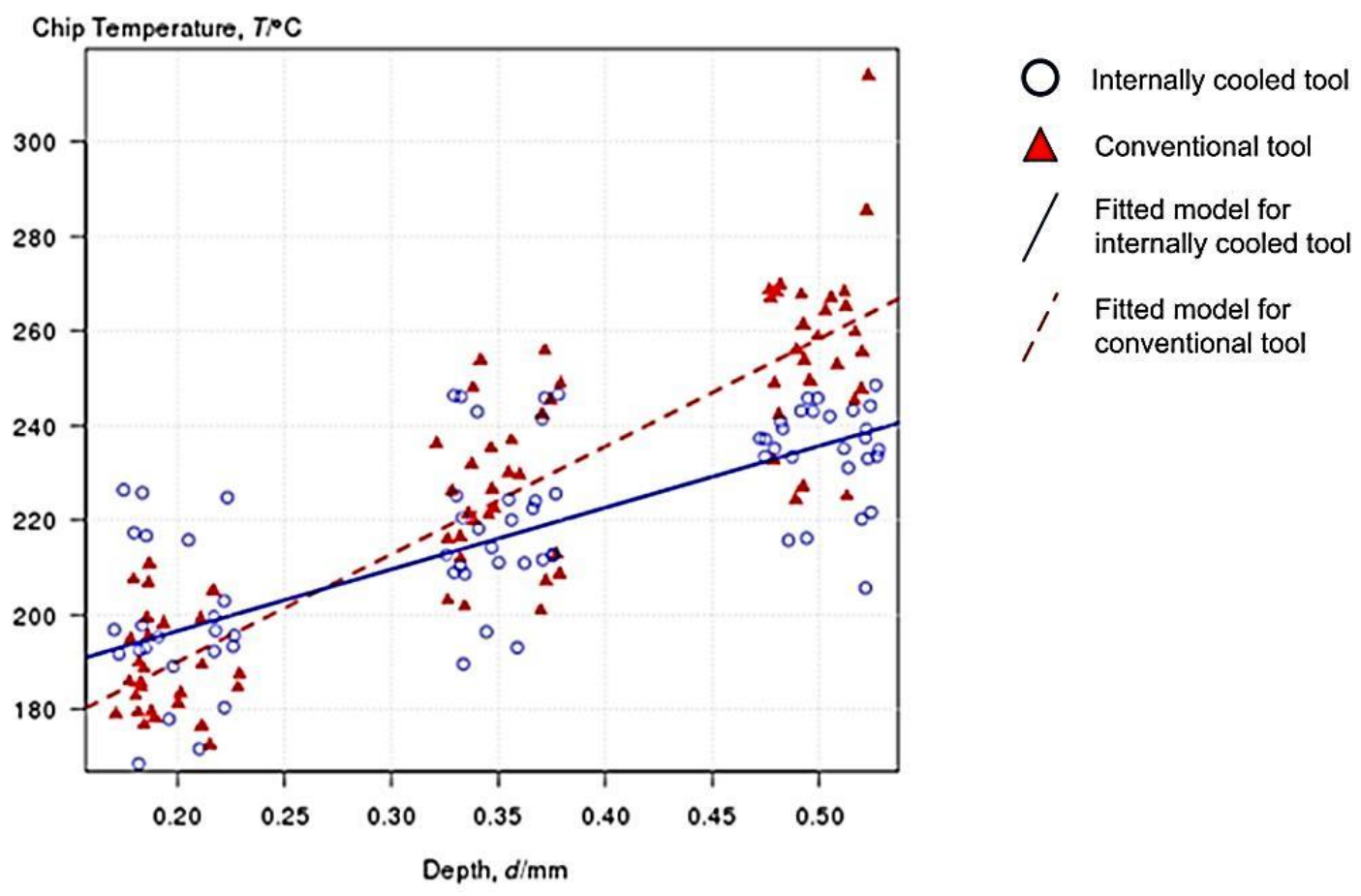

Figure 8. Back chip temperatures against depth of cut.

These continuous and dashed lines in Figure 8 represent the models fitted to the experimental results from the cutting trials with an internally cooled and a conventional tool, respectively. A possible different interpretation of the intersection between the two lines is that the internally cooled tool is indeed effective in reducing the chip temperature only beyond a critical depth of cut. Further investigation, which is outside the scope of this investigation, is required to clarify this point.

\section{CONCLUSIONS}

Chip temperature in turning AA6082-T6 aluminum alloy with conventional and internally cooled turning tools were compared in two separated $3 \times 3$ factorial experiments in the space of the technological variables: depth of cut, cutting speed, and feed rate. No external coolant was used in the machining trials. Linear statistical models with homoscedastic and heteroscedastic errors were fitted to the experimental results. To estimate the parameters of the fitted models, the ordinary least squares and restricted maximum likelihood methods were used, respectively. The statistical analyses showed that the measured chip temperature appears to depend significantly on the depth of cut only and not on the feed rate nor on the cutting speed. These analyses also suggest that as the depth of cut is increased, the internally cooled tool is more effective in reducing the chip temperature incrementally than the conventional tool is. Consequently, when no working metal fluids are used, internally cooled tools appear to be potentially advantageous over conventional tool in roughing operations.

\section{ACKNOWLEDGEMENTS}

This study was performed within the scope of the collaborative research project ConTemp, funded by the European Commission Seventh Framework Programme fp7 
(project number: NMP2-SL-2009-228585). The authors gratefully acknowledge the committed support of all the technical staff of the AMEE Department of Brunel University. Particular gratitude is expressed to Mr. Paul Yates.

\section{REFERENCES}

Bernstein, D. I., Lummus, Z. L., \& Santilli, G. (1995). Machine operator's lung a hypersensitivity pneumonitis disorder associated with exposure to metalworking fluid aerosols. CHEST Journal, 108(3), 636-641.

Byrne, G., Dornfeld, D., \& Denkena, B. (2003). Advancing cutting technology. CIRP Annals-Manufacturing Technology, 52(2), 483-507.

Crawley, M. J. (2012).The R book. NY: John Wiley.

Davies, M. A., Ueda, T., \& M'Saoubi, R. (2007). On the measurement of temperature in material removal processes. CIRP Annals - Manufacturing Technology, 56(2), 581-604.

Dinc, C., Lazoglu, I., \& Serpenguzel, A. (2008). Analysis of thermal fields in orthogonal machining with infrared imaging. Journal of Materials Processing Technology, 198(1), 147-154.

Faraway, J. J. (2005). Extending the linear model with $R$ (Texts in Statistical Science).

Jeffries, N. P. (1972). Internal cooling of metal-cutting tools. Industrial Lubrication and Tribology, 24(4), 179-181.

Jeffries, N., \& Zerkle, R. (1970). Thermal analysis of an internally-cooled metal-cutting tool. International Journal of Machine Tool Design and Research, 10(3), 381399.

Kadirgama, K., Rahman, M. M., Ismail, A. R., \& Bakar, R. A. (2011). Finite element analysis of HASTELLOY C-22HS in end milling. Journal of Mechanical Engineering and Sciences, 1, 37-46.

Khan, M. A. R., Rahman, M. M., Kadirgama, K., Maleque, M. A., \& Ishak, M. (2011). Prediction of surface roughness of Ti-6Al-4V in electrical discharge machining: a regression model. Journal of Mechanical Engineering and Sciences, 1, 16-24.

Klocke, F., \& Eisenblätter, G. (1997). Dry cutting. CIRP Annals-Manufacturing Technology, 46(2), 519-526.

Lazoglu, I., \& Altintas, Y. (2002). Prediction of tool and chip temperature in continuous and interrupted machining. International Journal of Machine Tools and Manufacture, 42(9), 1011-1022.

Longbottom, J., \& Lanham, J. (2005). Cutting temperature measurement while machining-a review. Aircraft Engineering and Aerospace Technology, 77(2), 122-130.

McGill, R., Tukey, J. W., \& Larsen, W. A. (1978). Variations of box plots. The American Statistician, 32(1), 12-16.

Najiha, M. S., Rahman, M. M., Yusoff, A. R., \& Kadirgama, K. (2012a). Investigation of flow behavior in minimum quantity lubrication nozzle for end milling processes. International Journal of Automotive and Mechanical Engineering, 6, 768-776.

Najiha, M. S., Rahman, M. M., Kamal, M., Yusoff, A. R., \& Kadirgama, K. (2012b). Minimum quantity lubricant flow analysis in end milling processes: a computational fluid dynamics approach. Journal of Mechanical Engineering and Sciences, 3, 340-345. 
O'Sullivan, D., \& Cotterell, M. (2001). Temperature measurement in single point turning. Journal of Materials Processing Technology, 118(1-3), 301-308.

Pinheiro, J. C., \& Bates, D. M. (2000). Mixed effects models in S and S-PLUS. New York: Springer Verlag.

Pinheiro, J., Bates, D., \& DebRoy, S. (2007). Linear and nonlinear mixed effects models. R Package Version, 3: 57.

Quan, Y., He, Z., \& Dou, Y. (2008). Cutting heat dissipation in high-speed machining of carbon steel based on the calorimetric method. Frontiers of Mechanical Engineering in China, 3(2), 175-179.

RDevelopment, C. (2011). TEAM. 2008. R: A Language and environment for statistical computing. $R$ foundation for statistical computing. Vienna, Austria.

Rozzi, J. C., Sanders, J. K., \& Weibo, C. (2011). The experimental and theoretical evaluation of an indirect cooling system for machining. Journal of Heat Transfer, 133(3), 031006, 1-10.

Sanchez, L. E., Scalon, V. L., \& Abreu, G. G. (2011). Cleaner machining through a toolholder with internal cooling. Proceedings of third international workshop on advances in cleaner production, São Paulo, Brazil, May, Anonymous pp. 18-20.

Singh, R., \& Singh, B. (2011). Comparison of cryo-treatment effect on machining characteristics of titanium in electric discharge machining. International Journal fo Automotive and Mechanical Engineering, 3, 239-248.

Spitler, D., Lantrip, J., \& Nee, J. G. (2003). Fundamentals of tool design. Society of Manufacturing Engineers, Canada.

Sreejith, P., \& Ngoi, B. (2000). Dry machining: machining of the future. Journal of Materials Processing Technology, 101(1), 287-291.

Taylor, F. W. (1907). On the art of cutting metals. ASME Transactions, 28, 31-350.

Venables, W. N., Ripley, B. D., \& Venables, W. (1994). Modern applied statistics with $S$-PLUS. New York: Springer-Verlag.

Weinert, K., Inasaki, I. \& Sutherland, J. (2004). Dry machining and minimum quantity lubrication. CIRP Annals-Manufacturing Technology, 53(2), 511-537.

Zhao, H., Barber, G. \& Zou, Q. 2006. Effect of internal cooling on tool-chip interface temperature in orthogonal cutting. Tribology Transactions, 49(2), 125-134. 